\title{
Sclerosing Polycystic Adenosis
}

\author{
Colin A. Eliot $\cdot$ Alice B. Smith $\cdot$ Robert D. Foss
}

Received: 7 November 2011/Accepted: 3 December 2011/Published online: 20 December 2011

(C) Springer Science+Business Media, LLC (outside the USA) 2011

\begin{abstract}
A 25-year-old female presented with a welldefined, painless mass of 1 year's duration in the right parotid gland. MR imaging revealed a relatively well-circumscribed lesion involving the deep and superficial lobes of the right parotid gland. Histologic examination of the resection resulted in a diagnosis of sclerosing polycystic adenosis, a rare benign salivary gland entity of presumed non-neoplastic origin. The clinical, radiographic, and morphologic features of sclerosing polycystic adenosis are discussed.
\end{abstract}

Keywords Sclerosing polycystic adenosis .

Salivary gland · Parotid · Radiology ·

Magnetic resonance imaging MRI

\section{History}

A 25-year-old Caucasian female presented with a 1-year history of painless swelling in the right parotid gland.

Disclaimer The views expressed in this article are those of the author and do not necessarily reflect the official policy or position of the Department of the Navy, Army, Air Force, Department of Defense nor the U.S. Government.

\section{A. Eliot}

Naval Postgraduate Dental School, Bethesda, MD, USA

A. B. Smith

Uniformed Service University of the Health Sciences,

Bethesda, MD, USA

R. D. Foss $(\square)$

Department of Oral and Maxillofacial Pathology,

Naval Postgraduate Dental School, 8901 Wisconsin Avenue,

Bethesda, MD 20889, USA

e-mail: robert.foss@med.navy.mil
Clinical examination revealed a well-defined ovoid mass under the angle of the mandible. Fine needle aspiration biopsy of the lesion was non-diagnostic.

\section{Radiographic Features}

Magnetic resonance (MR) imaging revealed a relatively well-circumscribed lesion involving the deep and superficial lobes of the right parotid gland. The mass was low in signal on T1-weighted images (Fig. 1), and predominantly hyperintense on T2-weighted images with small cystic appearing areas (Fig. 2). After the administration of Gadolinium, fairly avid but heterogeneous enhancement was noted.

\section{Treatment}

A right parotidectomy was performed.

\section{Diagnosis}

Grossly, the superficial and deep parotid lobes contained multiple, discrete, firm white nodules measuring $4 \mathrm{~cm}$ in aggregate. On cut section, multiple cystic cavities were evident in the nodules. Histologic examination revealed effacement of the parotid parenchyma by a multinodular sclerosing process. Microscopically, the lesion consisted of circumscribed nodules of dense, hyalinized fibrous tissue containing a variety of epithelial elements. Epithelial components included variably sized cysts, degenerating acinar cells, and ductal adenosis (Fig. 3). Cystic structures were frequently lined by apocrine-like cells (Fig. 4), while 


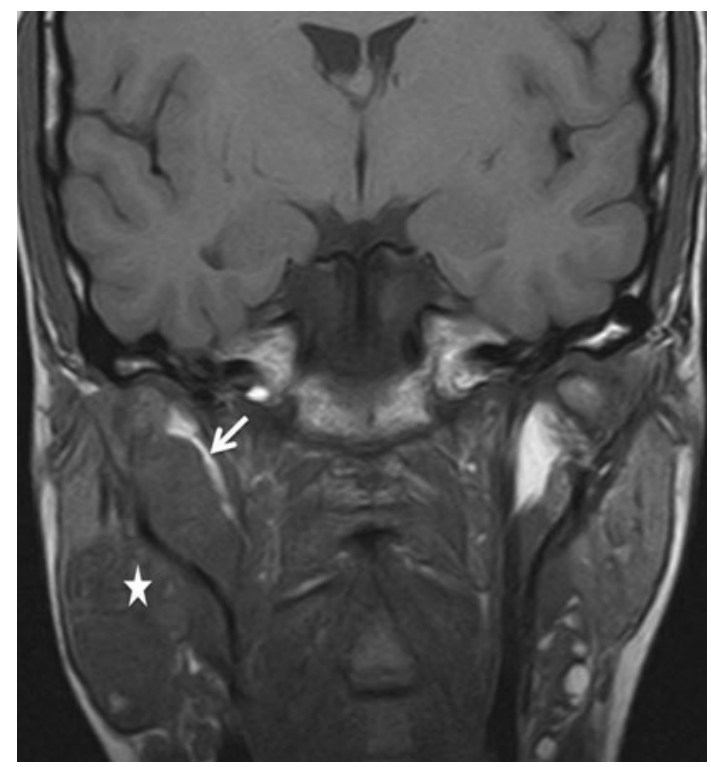

Fig. 1 Coronal T1-weighted image demonstrates a relatively wellcircumscribed, low signal lesion (star) that involves both the superficial and deep lobes of the right parotid gland. The portion involving the deep lobe extends to distort the high signal of the parapharyngeal fat (arrow)

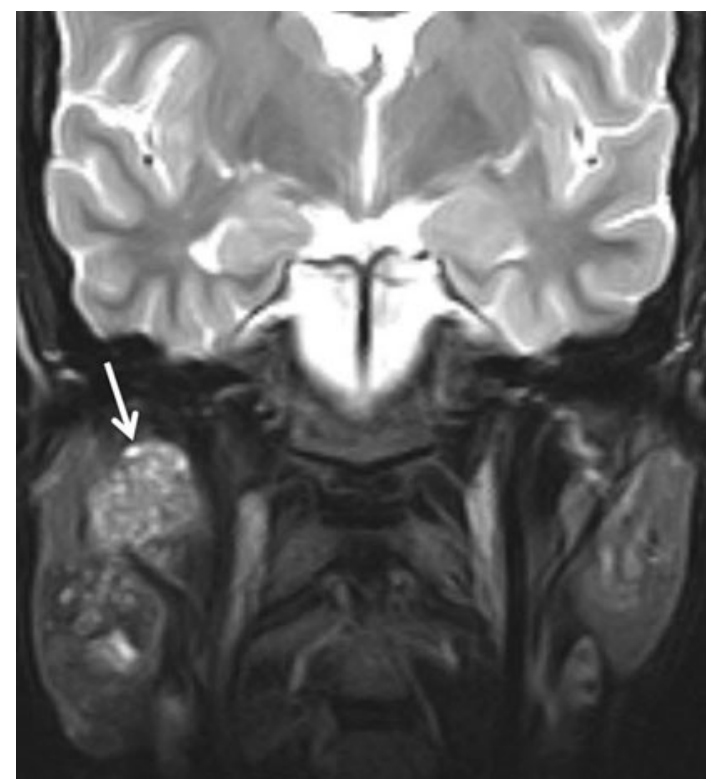

Fig. 2 Coronal T2-weighted image reveals a heterogeneous, predominantly hyperintense lesion with small high signal, cystic appearing foci (arrow)

coarse eosinophilic granules were present in altered acinar cells (Fig. 5). Rare ductal structures contained hyperplastic epithelium in a cribriform arrangement. In some microscopic fields, there was preservation of lobular salivary gland architecture or evidence of transition between normal and lesional tissue. Additional findings included xanthomatous replacement of ductal epithelium and a sparse

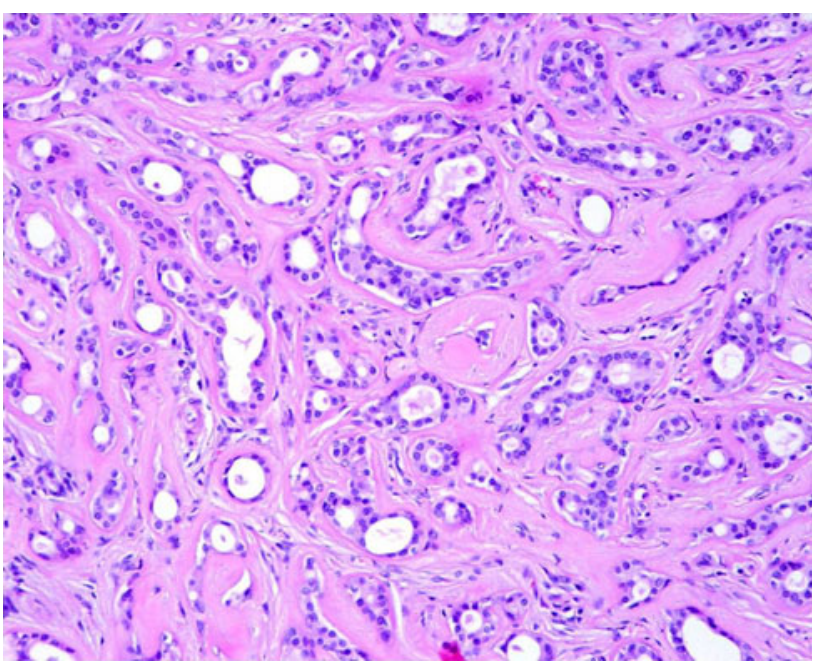

Fig. 3 The epithelial components of the lesion include zones of prominent ductal adenosis

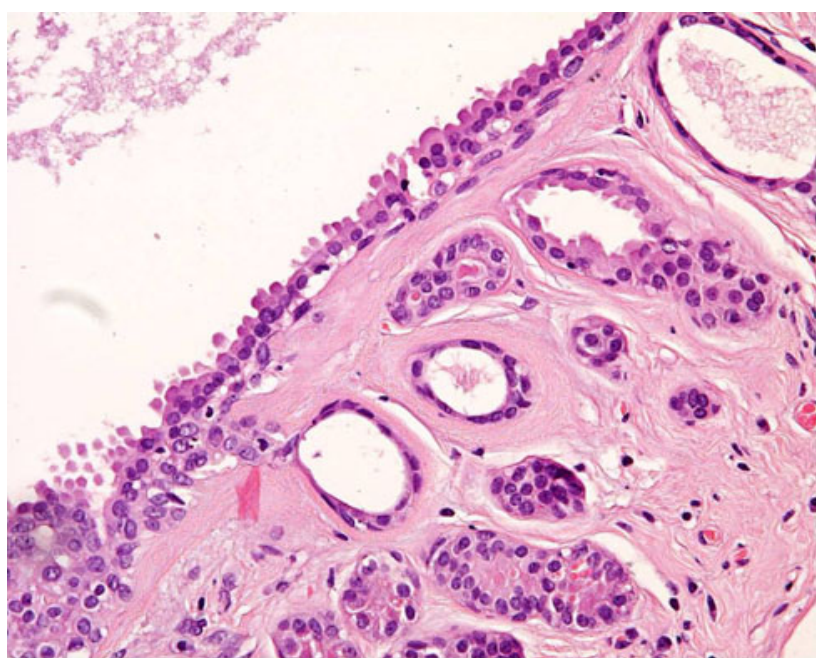

Fig. 4 Cystic structures are frequently lined by apocrine-like cells

lymphoplasmacytic inflammatory infiltrate. The histomorphologic features were characteristic of sclerosing polycystic adenosis (SPA).

\section{Discussion}

SPA is a rare, benign epithelial salivary gland entity first described by Smith, et al. [1]. The pathogenesis of SPA is unknown. While initially described as a reactive, postinflammatory process, one recent study has addressed a possible association with Epstein-Barr virus, however additional confirmatory studies are needed [1-3]. In addition, there is evidence that monoclonal cell populations exist in some cases of SPA, although the significance of this clonality is uncertain [4]. SPA has been reported in a 


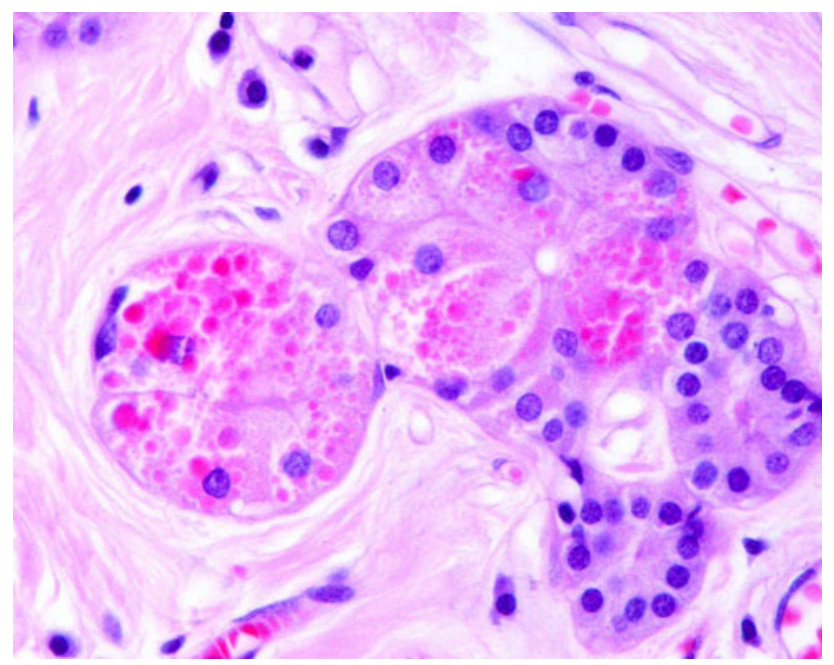

Fig. 5 Characteristic coarse, eosinophilic granules are present within altered acinar cells

wide age range of patients from childhood to the eighth decade of life with case series reporting mean ages of 28-44.5 years [1,2]. Men and women are affected with approximately equal frequency, although a slight female predilection has been suggested [2]. SPA is characteristically a lesion of the major salivary glands, with the majority of cases involving the parotid gland, but minor salivary gland examples have been reported [1-3]. The typical presenting complaint is of a slow-growing, asymptomatic swelling in the affected gland. Occasionally, mild pain or tingling may be present [1]. There have only been limited published descriptions of the imaging findings in SPA due to the rarity of the lesion. It has been described as a parotid mass with small cystic areas that are high in signal intensity on $\mathrm{T} 2$-weighted $\mathrm{MR}$ imaging with enhancement on post-contrast imaging. On ultrasound, it is described as a hypoechogenic, well-circumscribed lesion with microcystic areas [5-7]. Grossly, SPA appears as circumscribed, pale, rubbery nodules embedded in normalappearing salivary gland parenchyma $[1,2]$. The nodules may range from 1 to $12 \mathrm{~cm}$ in diameter and the lesion may present as a multinodular mass. Microscopically, SPA is composed of circumscribed lobules of hyalinized connective tissue. Within the fibrous tissue is hyperplastic epithelium with ductal and acinar differentiation. There are ectatic ducts and cysts with apocrine-like metaplasia and epithelial hyperplasia that may exhibit cribriform growth $[1,8]$. Atypia may be found within the ductal epithelium to a degree that warrants the diagnosis of dysplasia [9]. Occasional hyaline globules reminiscent of collagenous spherulosis may be present. Coarse, strongly eosinophilic cytoplasmic granules are often found within acinar structures $[1,2]$. The features of SPA are similar to fibrocystic changes of the breast and some evidence suggests that cells within SPA exhibit estrogen and progesterone receptor activity [9].

The primary histologic differential diagnostic considerations for SPA are polycystic (dysgenetic) disease (PDD) and sclerosing sialadenitis. PDD presents as a collection of cysts in a honeycombed lattice-like pattern; however, ductal or acinar epithelial proliferations are absent and fibrosis and inflammation are minimal. Conversely, sclerosing sialadenitis features a prominent fibrotic component with variable inflammation but lacks a cystic component and does not present in a multinodular fashion $[1,2]$.

SPA is considered amenable to conservative surgical excision with tumor-free margins. Facial nerve preservation is recommended for parotid lesions. Recurrence is rare and is most likely due to incomplete surgical excision or occult multifocal disease rather than true recurrence $[1,2,8]$.

Acknowledgments The authors wish to thank Drs. Rosario C. Gijón and Estafanía F. Delgado for contributing this case.

\section{References}

1. Smith BC, Ellis GL, Slater LJ, et al. Sclerosing polycystic adenosis of major salivary glands: a clinicopathologic analysis of nine cases. Am J Surg Pathol. 1996;20:161-70.

2. Gnepp DR, Wang LJ, Brandwein-Gensler M, et al. Sclerosing polycystic adenosis of the salivary gland. a report of 16 cases. Am J Surg Pathol. 2006;30:154-64.

3. Swelam WM. The pathogenic role of Epstein-Barr virus (EBV) in sclerosing polycystic adenosis. Pathol Res Pract. 2010;206:565-71.

4. Skálová A, Gnepp DR, Simpson RH, et al. Clonal nature of sclerosing polycystic adenosis of salivary glands demonstrated by using the polymorphism of the human androgen receptor (HUMARA) locus as a marker. Am J Surg Pathol. 2006;30:939-44.

5. Bharadwaj G, Nawroz I, O’Regan B. Sclerosing polycystic adenosis of the parotid gland. $\mathrm{Br} \mathrm{J}$ Oral Maxillofac Surg. 2007;45:74-6.

6. Etit D, Pilch BZ, Osgood R, et al. Fine-needle aspiration biopsy findings in sclerosing polycystic adenosis of the parotid gland. Diagn Cytopathol. 2007;37:444-7.

7. Fulciniti F, Losito NS, Ionna F, et al. Sclerosing polycystic adenosis of the partotid gland: a report of one case diagnosed by fine-needle cytology with in situ malignant transformation. Diagn Cytopathol. 2010;38:368-73.

8. Mackle T, Mulligan AM, Dervan PA, et al. Sclerosing polycystic sialadenopathy. Arch Otolaryngol Head Neck Surg. 2004;130:357-60.

9. Skálová A, Michal M, Simpson RH, et al. Sclerosing polycystic adenosis of parotid gland with dysplasia and ductal carcinoma in situ. Virchows Arch. 2002;440:29-35. 